\title{
ULTRASOUND MULTIPATH BACKGROUND CLUTTER MITIGATION BASED ON SUBSPACE ANALYSIS AND PROJECTION
}

\author{
Shen Xizhong ${ }^{1}$, Yimin D. Zhang ${ }^{2}$, Moeness G. Amin ${ }^{2}$, Ramazan Demirli ${ }^{2}$ \\ ${ }^{1}$ Electrical and Automatic School, Shanghai Institute of Technology, China \\ ${ }^{2}$ Center for Advanced Communications, Villanova University, USA \\ shen_sh@yahoo.cn; \{yimin.zhang; moeness.amin; ramazan.demirli\}@villanova.edu
}

\begin{abstract}
In this paper, we consider ultrasound imaging of flaws in a metallic alloy where the presence of strong bottom surface reflection and other interference signals constitutes a challenging problem. A subspace-based approach is developed for removing, or significantly reducing, bottom surface reflections to enhance ultrasound imaging. In constructing the surface reflection, or clutter, subspace, we account for rough surface scatterings which, due to various possible propagation time delays between the transmitter and receiver, expand the subspace dimension beyond that corresponding to ideal propagation. We also estimate and compensate, using signal correlation methods, for changes in the same time delays due to imperfect sensor displacements on the top surface of the alloy. Experimental results show that substantial clutter suppression can be achieved with negligible effects to the flaw signals.
\end{abstract}

Index Terms - Ultrasound imaging, clutter suppression, subspace projection

\section{INTRODUCTION}

Ultrasound nondestructive testing (NDT) provides reliable and efficient structure evaluation and machine status monitoring in aerospace, automotive, and other transportation sectors as well as many civil and mechanical engineering applications [1-2]. A very challenging problem encountered in ultrasound imaging is the existence of strong background clutter, caused by bottom and/or side surface reflections, inhomogeneous material, and various propagation modes. Ultrasound clutter often overshadows the return signal from weak flaws, especially when the flaws are close to the surface. Significant efforts have been made to mitigate or remove the background clutter. For example, a sidelobe and clutter suppression method, called dual apodization with cross-correlation, is developed in [3] for contrast-to-noise ratio improvement in ultrasound imaging. In [4], eigen-based clutter filter designs are examined for the suppression of tissue clutter, which may be subject to motion, for ultrasound color-flow medical imaging.

Among different possible approaches to considerably attenuate clutter, direct subtraction of background response signal, measured in healthy references, from the response signal with flaw (target) is commonly used in radar signal processing [5]. Direct application of this method to practical ultrasonic imaging problems of metallic alloy, however, is difficult, owing to the roughness of the bottom surface and the thickness of the coupler (gel) between the transducers and the top surface. This roughness alters the delay profile of the bottom surface reflections and makes them dependent on transducer positions. Gating of bottom clutter in the time domain is an effective clutter suppression method, but it may also eliminate useful signal returns from flaws that are nearby the bottom surface of alloy.

This research is supported in part by National Science Foundation (NSF) under grant number IIP-0917690 and by The Ministry of Railways under grant number 2011J011-E.
In this paper, we examine the use of subspace processing and projection for the suppression of background clutter [4-8], which is also referred to as clutter herein. It is shown that the target, or flaw, subspace is different from the clutter subspace, due to differences in their respective delay profiles. In the underlying applications, clutter subspace is multi-dimensional which encompasses transducer position dependent scatterings, surface roughness, and material inhomogeneity. In essence, the subspace of the strong clutter is estimated from different transducer locations and it corresponds to the singular vectors of the dominant singular values of the data observation matrix. In addition to the bottom surface scatterings, we also estimate and compensate for changes in the time delays due to imperfect sensor displacements on the top surface of the alloy, using signal correlation. As such, robust clutter subspace can be constructed with low rank energy concentration. Orthogonal clutter subspace projection, which is insensitive to magnitude and phase ambiguities, is applied to increase the signal-to-clutter ratio and deal with multiple clutter components. Real measurements as well as synthetic data are used in the evaluation of the proposed technique. For increased degree of flaw signal protection, the multi-bouncing clutters from top and bottom surfaces are incorporated in the clutter subspace construction so as to add more distinction between the flaw signal and the clutter signatures. The results show that a substantial clutter suppression is achieved with negligible effect to the flaw signal. This implies that the flaw signal subspace is different from the clutter subspace which is attributed to differences in their corresponding time-delay profiles.

\section{SIGNAL MODEL}

Consider a multiple-input multiple-output (MIMO) ultrasonic imaging system consisting of $M$ transmit transducers and $N$ receive transducers. A return signal received at the $n$-th receive transducer, $r_{n}(t)$, is modeled as a linear combination of the transmitted signal with different delays, i.e.,

$$
r_{n}(t)=\sum_{m=1}^{M} \sum_{i} \beta_{i, n, m} s_{m}\left(t-\tau_{i, n, m}\right),
$$

where $s_{m}(t)$ is the emitted signal from the $m$-th transmit transducer, whereas $\beta_{i, n, m}$ and $t_{i, n, m}$ are, respectively, the reflectivity coefficient and delay of the $i$-th propagation path. In practice, ultrasound returns may have other responses that are not accounted in model (1). The consideration of these components, however, is outside of the scope of this paper.

Assuming that signals corresponding to the different transmit transducers are separable through orthogonal waveform design utilizing the time, frequency, and/or code dimensions (see for example [9]), we can isolate the received signal corresponding to the $m$-th transmit transducer and the $n$-th receive transducer,

$$
r_{n, m}(t)=\sum_{i} \beta_{i, n, m} s_{m}\left(t-\tau_{i, n, m}\right)
$$


Ideally, for a homogeneous alloy with perfectly smooth surface, the bottom surface scattering assumes perfect mirror reflection. In practice, however, there could be roughness in the material surface due to machining inaccuracy or other reasons, yielding scattering and changes in the propagation delays. In essence, for some paths, the angles of reflection are not equal to the angles of incidence on the bottom surface of the alloy (see Fig. 1(a)). As such, there exist multiple paths between any pair of transducers which underline the summation in Eq. (2). In addition, material inhomogeneity may alter the propagation velocity. Due to the small differences in the corresponding path lengths and propagation velocities, the time delay, $\tau_{i, n, m}$ described in Eq. (2), can be cast as perturbation of the mean value. Additionally, it is recognized that there might be small gaps between the ultrasound transducers and the surface, where coupler may be partly or fully filled with, as depicted in Fig. 1(b). These gaps contribute to the group delay time, $\Delta \tau_{\text {group }, n, m}$, and shift the entire return signal for each transmitter-receiver combination.

With the consideration of the above two perturbation factors of the time delay, Eq. (2) becomes

$$
r_{n, m}(t)=\sum_{i} \beta_{i, n, m} s_{m}\left(t-\tau_{i n, m}-\Delta \tau_{\text {group }, n, m}-\Delta \tau_{i, n, m}\right) .
$$

Because the focus in this paper is the removal of clutter, we consider a single transmitter-receiver pair and thus subscripts $n$ and $m$ are omitted from the expression for notational simplicity.
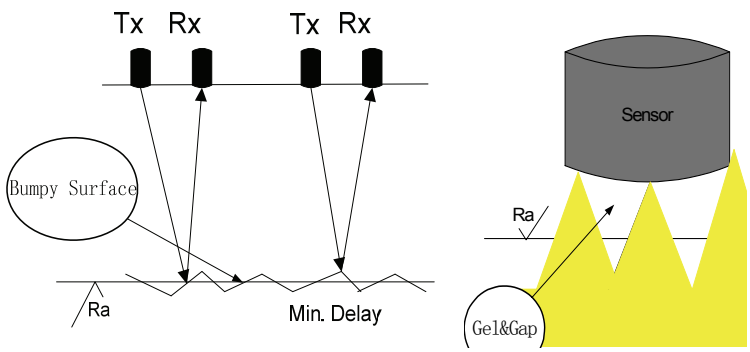

(a) Roughness at reflection surface (b) Roughness around sensors

Fig. 1. Illustration of delay spread due to surface roughness.

\section{ULTRASOUND MULTIPATH BACKGROUND CLUTTER MITIGATION STRATEGY}

\subsection{Background Clutter Mitigation}

Consider a simple scenario that uses a metallic alloy as the subject under test. A measured signal, $r(t)$, includes two components, flaw reflection, $r_{F}(t)$, and background clutter, $r_{B}(t)$. With the consideration of measurement noise $v_{n}(t), r(t)$ can be described by

$$
r(t)=r_{F}(t)+r_{B}(t)+v_{n}(t) .
$$

In the above expression, the measurement noise $v_{n}(t)$ typically follows zero-mean independent Gaussian distribution. Each component of $r_{F}(t)$ and $r_{B}(t)$ can be characterized using the model depicted in Eq. (3). Measurement noise is usually not considered as a significant factor since its effect can be mitigated by averaging over multiple observations.

To reveal multipath flaw echoes that may be overshadowed by the background clutter, the objective is to remove or mitigate the background clutter without significantly affecting the flaw signal. Construct a set of reference signal bases, $u_{k}(t), k=1, \ldots, K$, which represent the clutter, where $K$ is the number of clutter bases. Then, clutter removal can be achieved as

$$
\hat{r}_{F}(t)=r(t)-\sum_{k=1}^{K} \alpha_{k} u_{k}(t)
$$

where $\alpha_{k}$ is the $k$-th coefficient that minimizes the norm of the above residual difference. To account for varying clutter responses as well as delay profiles, the clutter subspace is constructed using $L$ measurement data, $r_{(}(t), l=1, \ldots, L$, in the absence of flaw, observed at $L$ different positions of the transducer pair. The distance between the transmitter and receiver is kept the same. This type of measurements can be performed in a healthy object before it is actually used, or by measuring a healthy identical replica. Ideally, the clutter profiles should be identical, assuming homogeneous propagation medium. As we discussed earlier, however, scattering from a rough surface may cause them to differ. To take the delay spread into account, two pre-processing steps, respectively described in the following two subsections, are performed before the clutter signal subspace processing.

\subsection{Observation Alignment through Correlation}

To compensate for the group delay $\Delta \tau_{\text {group }, l}$ for the $l$-th measurement, we apply correlation between the $l$-th observed signal (with or without flaw), $r_{l}(t)$, and a reference signal, $r_{\text {ref }}(t)$, which is measured in the absence of flaw signal. That is,

$$
\Delta \tau_{\text {group }, l}=\arg \max _{\tau}<r_{l}(t-\tau), r_{\text {ref }}(t)>,
$$

where $\langle>$ denotes the mean of the inner-product of its elements. Once $\Delta \tau_{\text {group }, l}$ is obtained, the clutter responses can be aligned and the effect of such group delays is minimized.

As the signals are collected and processed using discrete-time representations, the group delay is expressed in terms of the number of samples, $D_{\text {group }, l}=\left\lceil\Delta \tau_{\text {group }, l} f_{s}\right\rceil$, where $\lceil x\rceil$ denotes nearest integer greater than or equal to $x$, and $f_{s}$ is the sampling rate. Note that, in practice, ultrasound signals are usually oversampled far beyond the Nyquist rate to achieve improved ultrasound image quality. As a result, the discrete signal representation of the $l$-th received clutter response, after signal alignment, is represented as a vector $\mathbf{r}_{l}^{(0)}=\left[r_{l}[1], \ldots, r_{l}[T]\right]^{T}$, where $T$ is the total number of data samples used in the vector, whereas $(.)^{T}$ denotes vector or matrix transpose. Superscript $(0)$ denotes the fact that no artificial delays, as discussed below, are applied.

\subsection{Subspace Dimension Expansion}

For effective clutter removal, all possible values of the local delay, $\Delta \tau_{l}$, should be taken into account in clutter subspace construction. That is, consider a clutter response $r_{l}(t)$, all of its delayed variations, $r_{l}(t-\Delta \tau)$, up to the maximum potential extent of the delay spread, i.e., $\Delta \tau \in\left[-\Delta \tau_{\max }, \Delta \tau_{\max }\right]$, should be taken into account.

Using the discrete-time signal representations, the above discussion implies that, in addition to vector $\mathbf{r}_{l}^{(0)}=\left[r_{l}[1], \ldots, r_{l}[T]\right]^{T}$, the vectors that account for delayed copies $\mathbf{r}_{l}^{(d)}=\left[r_{l}[d+1], \ldots, r_{l}[T+d]\right]^{T}$ should also be considered as possible received clutter signals for $-D \leq d \leq D$ (proper zeropadding is assumed for time instants that are out of the measurement range).

\subsection{Subspace Construction}

Now, we construct the following matrix, $\mathbf{B}$, for subspace analysis from $L$ measurements,

$$
\mathbf{B}=\left[\mathbf{r}_{1}^{(-D)}, \mathbf{r}_{1}^{(-D+1)}, \ldots, \mathbf{r}_{1}^{(D)}, \ldots, \mathbf{r}_{L}^{(-D)}, \mathbf{r}_{L}^{(-D+1)}, . ., \mathbf{r}_{L}^{(D)}\right] .
$$

The dimension of matrix $\mathbf{B}$ is $T \times(2 D+1) L$. We assume that $T \gg(2 D+1) L$, which is typically satisfied in practice, so that the maximum rank of the clutter signal subspace is $(2 D+1) L$. Applying 
singular value decomposition (SVD) to matrix $\mathbf{B}$ yields [10]

$$
\mathbf{B}=\mathbf{U} \boldsymbol{\Sigma} \mathbf{V}^{T}=\left[\begin{array}{ll}
\mathbf{U}_{B} & \mathbf{U}_{B}^{\perp}
\end{array}\right]\left[\begin{array}{cc}
\boldsymbol{\Sigma}_{B} & \mathbf{O} \\
\mathbf{O} & \boldsymbol{\Sigma}_{B}^{\perp}
\end{array}\right]\left[\begin{array}{c}
\mathbf{V}_{B}^{T} \\
\left(\mathbf{V}_{B}^{\perp}\right)^{T}
\end{array}\right],
$$

where $\mathbf{U}=\left[\mathbf{u}_{1}, \ldots, \mathbf{u}_{(2 D+1) L}\right]$ and $\mathbf{V}=\left[\mathbf{v}_{1}, \ldots, \mathbf{v}_{(2 D+1) L}\right]$ are unitary matrices, containing the left and right character vectors, respectively, whereas the diagonal matrix $\Sigma$ contains the singular values. Matrices $\mathbf{U}$ (respectively $\mathbf{V}$ ) can each be divided into two parts, i.e., $\mathbf{U}_{B}$ and $\mathbf{U}_{B}^{\perp}$ (and respectively $\mathbf{V}_{B}$ and $\mathbf{V}_{B}^{\perp}$ ), which respectively span the signal subspace and noise subspace. Because no flaw signal is included in matrix $\mathbf{B}$, it is desirable to treat a large number of columns as the signal subspace of the clutter.

\subsection{Clutter Removal}

When the signal subspace of the clutter is properly identified, the removal of the clutter from a measured signal, in the presence of flaw reflection, can be achieved by projecting the received signal onto the noise subspace of the clutter. Consider a received signal $r(t)$ as described in Eq. (4) which includes both flaw signal and background clutter. The group delay $\Delta \tau_{\text {group }}$ of $r(t)$ can be estimated and compensated by using the same procedure as described in Section 3.2, where $r_{l}(t)$ is replaced by $r(t)$. A $T$-sample vector $\mathbf{r}$ is obtained from data observations after alignment.

To obtain the flaw signal vector, $\mathbf{r}_{F}$, i.e., the clutter-suppressed version of $\mathbf{r}$, we perform

$$
\widetilde{\mathbf{r}}_{F}=\left(\mathbf{I}-\mathbf{U}_{B} \mathbf{U}_{B}^{T}\right) \mathbf{r} .
$$

Obviously, this is equivalent to Eq. (5) by considering the columns of $\mathbf{U}_{B}$ as the clutter bases, whereas the elements of $\mathbf{U}_{B}^{T} \mathbf{r}$ as the respective coefficients.

\section{EXPERIMENTAL STUDY}

\subsection{Experiment Settings}

A block of aluminum alloy type 6061, illustrated in Fig. 2, was used in the experiments. The height of the alloy is $76.2 \mathrm{~mm}$. A flat bottom hole with a diameter of $3 \mathrm{~mm}$ and a depth of $21 \mathrm{~mm}$ was drilled into the material from the bottom to emulate a flaw.

Signal generation and measurement are performed using an Olympus Panametrics pulser, model 5072PR, operated in the monostatic (T/R) mode [11]. An Olympus transducer, model V-110M, with a center frequency of $5 \mathrm{MHz}$, is used. The sampling rate is 40 $\mathrm{MHz}$. Ten data sets are measured in the absence of a flaw, whereas one data set is measured in the presence of the flaw. The latter is depicted in Fig. 3. The flaw reflection is observed around $t=17.5$ $\mu \mathrm{s}$, whereas a smaller multipath signal appears in $41.4 \mu \mathrm{s}$ (see [12] for the details of multipath modes). The bottom reflections appear at $24.1 \mu$ s and its multiples.

\subsection{Singular Values}

Fig. 4 shows the distribution of the singular values of the reference matrix B expressed in Eq. (7). Fig. 4(a) is for matrix B consisting of only the 10 measured data $(D=0)$, where one singular value is dominant, followed by other 9 smaller but nonzero ones. In Fig. 4(b), expanded matrix $\mathbf{B}$ with $D=10$ is considered, yielding a total of 210 singular values. It is evident that dimension expansion of matrix $\mathbf{B}$ via the inclusion of delay shifted versions of measured signal significantly increases the dimensionality of the clutter subspace.

\subsection{Clutter Subspace with Delay Shifts}

To examine the effect of the inclusion of delay shifted version of the clutter in the estimation of clutter signal subspace, Fig. 5 compares the estimated flaw signal as the result of projection of the observations, which are shown in Fig. 3, onto the orthogonal clutter subspace, in two different scenarios. Fig. 5(a) shows the results based only on the observations (i.e., $D=0$ ), whereas Fig. 5(b) is obtained when a maximum delay of 10 samples $(D=10)$ is used. It is clear that the non-delay case yields a high clutter residual, whereas the clutter is almost completely removed when delay shifted versions of the clutter are considered in the construction of the clutter subspace.

\subsection{Detection of Flaw Close to Bottom Surface}

In this example, we consider the situation where a flaw is very close to the bottom surface. Due to machining difficulty, we synthetically add flaw data, which was originally measured from a circular hole at a different position, to the received signal depicted in Fig. 3. The standoff distance between the added flaw and the bottom surface is assumed to be $1 \mathrm{~mm}$. Fig. 6(a) shows the signal in the presence of the actual flat bottom hole and the artificial flaw, and Fig. 6(b) shows the signal after orthogonal projection. It is evident that the background surface echoes are greatly mitigated, and the flaw close to the surface is revealed after clutter removal.

\section{CONCLUSIONS}

We have considered the removal of ultrasonic background clutter for ultrasonic imaging of flaws in a metallic object. A signal model was established based on the surface microstructure concept. This clutter is caused by surface reflections and was removed by projecting the received signal onto a subspace that is orthogonal to the clutter subspace. Clutter subspace construction utilized multipath information from a healthy sample and incorporated perturbations in propagation delays, due to surface roughness, as a function of transducer position. In addition, cross-correlation techniques were used to align the clutter signal delays due to differences in transducer closeness to the top surface. The results show that substantial clutter suppression can be achieved with negligible effect on the flaw signal.

\section{REFERENCES}

[1] L. W. Schmerr and S.J. Song, Ultrasonic Nondestructive Evaluation Systems, Springer, 2007.

[2] R. Lasser, M. Lasser, J. Kula, D. Eich, and R. Westernik, "Remote, simple nondestructive testing of composite defects with an ultrasound camera: a Boeing study," in Proc. Composites, Las Vegas, NV, Feb. 2010.

[3] C. H. Seo and J. T. Yen, "Sidelobe suppression in ultrasound imaging using dual apodization with cross-correlation," IEEE Trans. Ultrasonics, Ferroelectrics, and Frequency Control, vol. 55, no. 10, pp. 2198-2210, Oct. 2008.

[4] A. C. H. Yu and L. Lovstakken, "Eigen-based clutter filter design for ultrasound color flow imaging: a review," IEEE Trans. Ultrasonics, Ferroelectrics, and Frequency Control, vol. 57, no. 5, pp. 1096-1111, May 2010.

[5] A. M. Mayordomo and A. Yarovoy, "Optimal background subtraction in GPR for humanitarian demining," in Proc. European Radar Conf., Amsterdam, Netherlands, pp. 48-51, Oct. 2008.

[6] V. Kovalenko, A. G. Yarovoy, and L. P. Ligthart, "A novel clutter suppression algorithm for landmine detection with GPR," IEEE Trans. Geoscience and Remote Sensing, vol. 45, no. 11, Nov. 2007.

[7] C. Debes, C. Weiss, A. M. Zoubir and M. G. Amin, "Wall clutter mitigation using cross-beamforming in through-thewall radar imaging," in Proc. EUSIPCO, Aalborg, Denmark, pp. 1097-1100, Aug. 2010. 
[8] F. H. C. Tivive, A. Bouzerdoum, and M. G. Amin. "An SVDbased approach for mitigating wall reflections in through-thewall radar imaging," in Proc. IEEE Radar Conf., Kansas City, KS, May 2011.

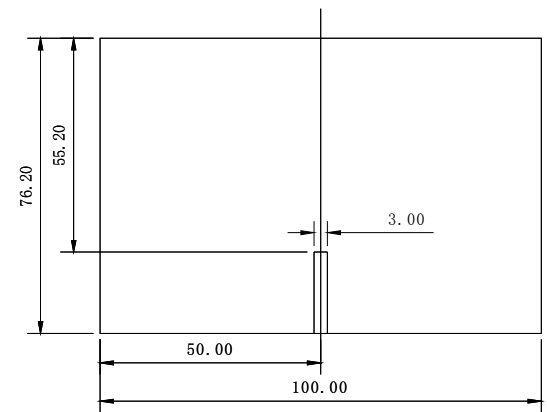

Fig. 2 Dimension of the aluminum alloy and the flat bottom hole (in $\mathrm{mm}$ ).
[9] J. Li and P. Stoica, MIMO Radar Signal Processing, Wiley, 2009.

[10] R. A. Horn and C. R. Johnson, Topics in Matrix Analysis, Cambridge Univ. Press, 1991.

[11] http://www.olympus-ims.com/en/5072pr/.

[12] X. Shen, Y. D. Zhang, R. Demirli, and M. G. Amin, "Ultrasonic flaw imaging exploiting multipath information," in Proc. SPIE, vol. 8064, Orlando, FL, April 2011.

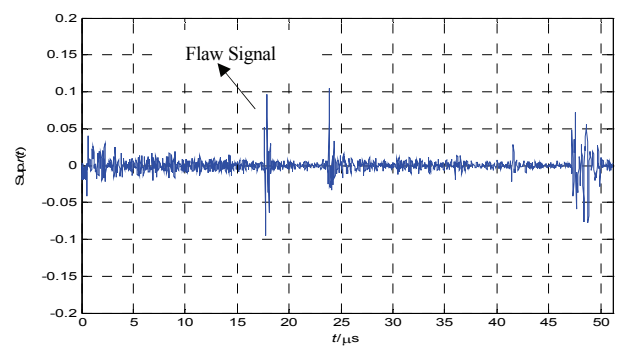

(a) with no shifted versions $(D=0)$

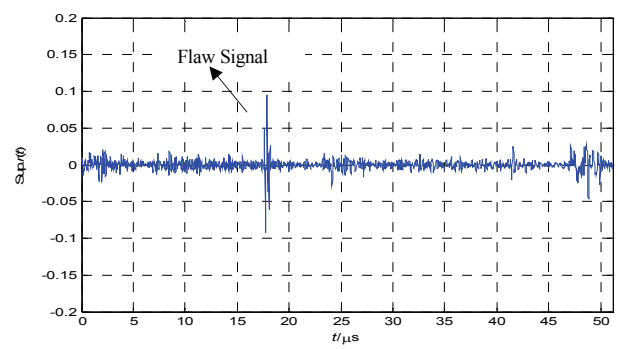

(a) with shifted versions $(D=10)$

Fig. 5 Background mitigation with different delay shifts.

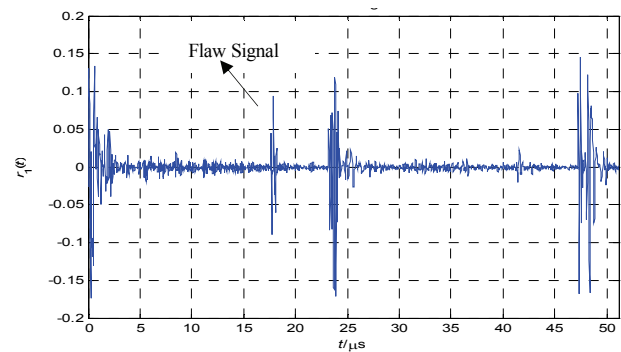

(a) before clutter removal

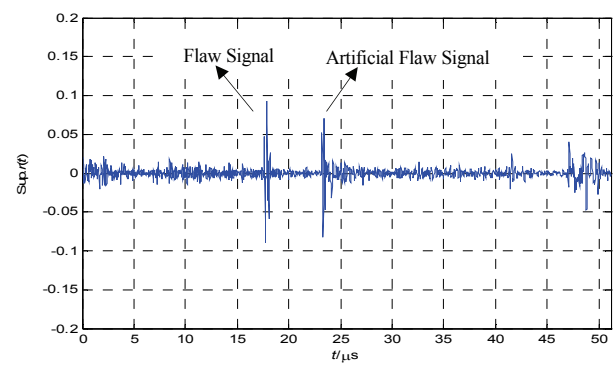

(b) after clutter removal

Fig. 6 Flaw with $1 \mathrm{~mm}$ standoff distance from the bottom surface.

Fig. 4 Singular value distribution in decreasing order. 